\title{
MET overexpression assessed by new interpretation method predicts gene amplification and poor survival in advanced gastric carcinomas
}

\author{
Sang Y Ha ${ }^{1,7}$, Jeeyun Lee ${ }^{2,7}$, So Y Kang ${ }^{1}$, In-Gu Do ${ }^{3}$, Soomin Ahn ${ }^{1}$, Joon O Park ${ }^{2}$, \\ Won K Kang ${ }^{2}$, Min-Gew Choi ${ }^{4}$, Tae S Sohn ${ }^{4}$, Jae M Bae ${ }^{4}$, Sung Kim ${ }^{4}$, Minji Kim ${ }^{5}$, \\ Seonwoo Kim ${ }^{5}$, Cheol K Park ${ }^{1}$, Sai-Hong Ignatius $\mathrm{Ou}^{6}$ and Kyoung-Mee Kim ${ }^{1}$
}

${ }^{1}$ Department of Pathology, Samsung Medical Center, Sungkyunkwan University School of Medicine, Seoul, Korea; ${ }^{2}$ Department of Medicine, Division of Hematology-Oncology, Samsung Medical Center, Sungkyunkwan University School of Medicine, Seoul, Korea; ${ }^{3}$ Samsung Cancer Research Institute, Samsung Medical Center, Seoul, Korea; ${ }^{4}$ Department of Surgery, Samsung Medical Center, Sungkyunkwan University School of Medicine, Seoul, Korea; ${ }^{5}$ Biostatistics Unit, Samsung Medical Center, Sungkyunkwan University School of Medicine, Seoul, Korea and ${ }^{6}$ Department of Medicine, Division of Hematology-Oncology, Chao Family Comprehensive Cancer Center, University of California Irvine School of Medicine, Irvine, CA, USA

\begin{abstract}
The establishment of better selection criteria for identifying sub-populations that may benefit from treatment is a key aspect of the development and success of targeted therapy. To investigate methods for assessing MET overexpression in gastric cancer, we conducted immunohistochemistry using a new anti-Total MET monoclonal antibody in a single-institution cohort of $\mathbf{4 9 5}$ patients. As antibody is directed against a membranous and/or cytoplasmic epitope, two interpretation methods were used: (1) membranous and cytoplasmic and (2) membranous alone. In selected 120 cases, copy number gain and mRNA expression levels were measured using quantitative real-time PCR. Further in situ hybridization confirmed the presence of MET gene amplification. Among the $\mathbf{4 9 5}$ gastric cancers, simultaneous membranous and cytoplasmic overexpression of MET was found in 108 cases (21.8\%) and membranous alone overexpression was observed in 40 cases (8.1\%). The highest correlation was observed in membranous and cytoplasmic staining of MET: MET expression scores correlated significantly with high MET mRNA levels $(r=0.465, P<0.0001)$, increased copy number gain $(r=0.393, P=0.000002)$ and amplification of MET gene. Moreover, patients with MET overexpression showed shorter overall survival (HR, $1.781 ; 95 \% \mathrm{Cl}, 1.324-2.395 ; \boldsymbol{P}<0.001)$ and disease-free survival $(\mathrm{HR}, 1.765 ; 95 \% \mathrm{Cl}$, 1.227-2.541; $P=0.002$ ) compared with patients without MET overexpression. However, membranous overexpression of MET did not highly correlate with mRNA level $(r=0.274, P=0.002)$, copy number gain or survival $(P>0.05)$. We developed highly correlating interpretation methods of MET immunohistochemistry in gastric carcinomas. MET overexpression is an independent prognostic factor and could be a potential target and predictor of benefit for targeted therapy with MET inhibitors.

Modern Pathology (2013) 26, 1632-1641; doi:10.1038/modpathol.2013.108; published online 28 June 2013
\end{abstract}

Keywords: gene amplification; immunohistochemistry; MET; overexpression; prognosis

Gastric cancer is still the second most common cause of cancer-related death in the world. $^{1}$

Correspondence: Dr K-M Kim, MD, PhD, Department of Pathology, Samsung Medical Center, Sunkyungkwan University School of Medicine, \#50, Irwon-Dong, Gangnam-Gu, Seoul 135-710, Korea.

E-mail: kkmkys@skku.edu

${ }^{7}$ These authors contributed equally to this work.

Received 4 February 2013; revised 6 May 2013; accepted 11 May 2013; published online 28 June 2013
Recently, the addition of trastuzumab to standard cytotoxic chemotherapy demonstrated significant survival benefit in HER2-positive gastric carcinomas. ${ }^{2}$ Nevertheless, HER2 is positive in only a small portion of gastric cancer patients $(7-20 \%)$; thus, more precise molecular segmentation is urgently needed. ${ }^{3}$ One potential target in gastric cancer was identified as MET proto-oncogene (hepatocyte growth factor receptor) in preclinical studies., ${ }^{1,4}$

As mutations in the kinase domain of MET gene are almost lacking in gastric carcinomas, ${ }^{5}$ its 
activation has been mostly attributed to gene amplification. ${ }^{6-8}$ Although earlier Japanese reports described MET gene amplification in approximately $20 \%$ of gastric cancers by comparative genomic in situ hybridization or southern blot analysis, ${ }^{9-13}$ recent FISH analyses showed rare or no amplification in locally advanced gastric carcinomas. ${ }^{3,14}$ MET activation through copy number gain measured by quantitative real-time PCR, ${ }^{3,7,8,14,15} \mathrm{FISH}^{6,14}$ or silver in situ hybridization, ${ }^{16}$ and protein overexpression assessed by immunohistochemistry have been reported in gastric carcinomas. ${ }^{5,7,17-23}$ However, there is a wide discrepancy in the incidences and prognostic significances of MET activation in gastric cancer, ${ }^{3,5,7,8,14-24}$ and correlations between copy number gain and protein overexpression have been very poor. ${ }^{7,14,24}$ The establishment of selection criteria in identifying sub-populations that may benefit from treatment is a key aspect of further development of anti-MET monoclonal antibody (METMab) and hepatocyte growth factor/scatter factor monoclonal antibody treatment. ${ }^{25}$

Recently, striking results using the METMab in combination with an EGFR inhibitor (erlotinib) have been reported in patients with non-small cell lung cancer (OAM4558g trial). ${ }^{25}$ In that study, METMab increased progression-free survival in patients with high levels of MET expression by immunohistochemistry compared with the group receiving erlotinib alone. ${ }^{26}$ Improvement in overall survival has also been reported in patients with advanced gastric adenocarcinoma in which treatment with the hepatocyte growth factor/scatter factor monoclonal antibody AMG102 (also known as rilotumumab) combined with chemotherapy was compared with chemotherapy alone. ${ }^{27}$ Even in this study, the best response was observed in patients expressing a high level of MET in the tumor. ${ }^{26}$ Thus, both the study with METMab in non-small cell lung cancer and the study with AMG102 in gastric cancer highlight an essential requirement for patient stratification to ensure clinical benefit: high MET expression in cancer cells.

Immunohistochemistry is now widely available in most pathology laboratories, and a preferred method for identification of protein overexpression to identify patients who might benefit from treatment with a particular therapeutic product. ${ }^{28}$ To better characterize MET-positive gastric cancers and develop a sensitive predictor of benefit from METMab, we conducted immunohistochemistry with the same monoclonal primary antibody used for recent MET clinical trials. ${ }^{25,27}$

\section{Materials and methods}

\section{Patients}

The formalin-fixed, paraffin-embedded primary pT3 gastric carcinoma tissue blocks from 495 patients
(95 pN0, 97 pN1, 95 pN2, 115 pN3a and 93 pN3b) were retrieved. Four tissue cores $(0.6 \mathrm{~mm}$ diameter $)$ were sampled from the deep invasive front, both lateral sides, and the luminal surface area of the representative tumor block using AccuMax (Isu Abxis, Seoul, Korea). All patients had undergone curative surgical resection and extensive (D2) lymph node dissection, and the postoperative adjuvant treatment according to INT-0116 (Southwest Oncology Group-9008) was administered in all patients. ${ }^{29}$ The institutional review board at Samsung Medical Centre approved this retrospective study.

Clinicopathologic characteristics obtained from medical records included gender, age, tumor size, tumor location, histological type, recurrence, survival and disease-free survival.

\section{Immunohistochemistry for MET Protein}

MET protein expression was evaluated using CONFIRM anti-Total MET (SP44) rabbit monoclonal primary antibodies (Ventana Medical Systems, Tucson, AZ, USA) with a Ventana BenchMark XT automated slide processing system according to the manufacturer's protocol. Briefly, $4 \mu \mathrm{m}$ tissue sections were deparaffinized and rehydrated, and antigens were retrieved for $40 \mathrm{~min}$ in a citrate buffer ( $\mathrm{pH}$ 6.1) at $95{ }^{\circ} \mathrm{C}$. DAB was used as the chromogen, and the sections were counterstained with hematoxylin.

For controls, cell blocks from MET-amplified MKN45 and SNU5 cell lines and MET-non-amplified SNU1 cell lines were used. Two independent pathologists (SYH and KMK) with no prior knowledge of clinicopathological or molecular results evaluated the results. As SP44 antibody is directed against a membranous and/or cytoplasmic epitope present in human normal epithelial or tumor cells, two interpretation methods were adopted: (1) membranous and cytoplasmic and (2) membranous alone based on predominant staining patterns (Figure 1). Both membranous and cytoplasmic staining was scored as follows: 0, no reactivity or faint staining; $1+$, faint or weak staining; $2+$, moderate staining; $3+$, strong staining in $>10 \%$ of tumor cells. Membranous alone staining was scored by consensus recommendation on HER2 scoring for gastric carcinoma ${ }^{30}$ : 0 , no reactivity; $1+$, faint/barely perceptible membranous reactivity; $2+$, weak-to-moderate complete or basolateral membranous reactivity; $3+$, moderate-to-strong complete or basolateral membranous reactivity in $>10 \%$ of tumor cells. MET overexpression was defined as $2+$ or $3+$ by membranous and cytoplasmic interpretation and only $3+$ by membranous interpretation.

In all cases with $3+$ expression either by membranous and cytoplasmic staining or by membranous staining alone, two pathologists agreed perfectly. In a few cases with $1+$ or $2+$ score, there was disagreement and the final interpretation was determined by consensus using the multi-head microscope. 


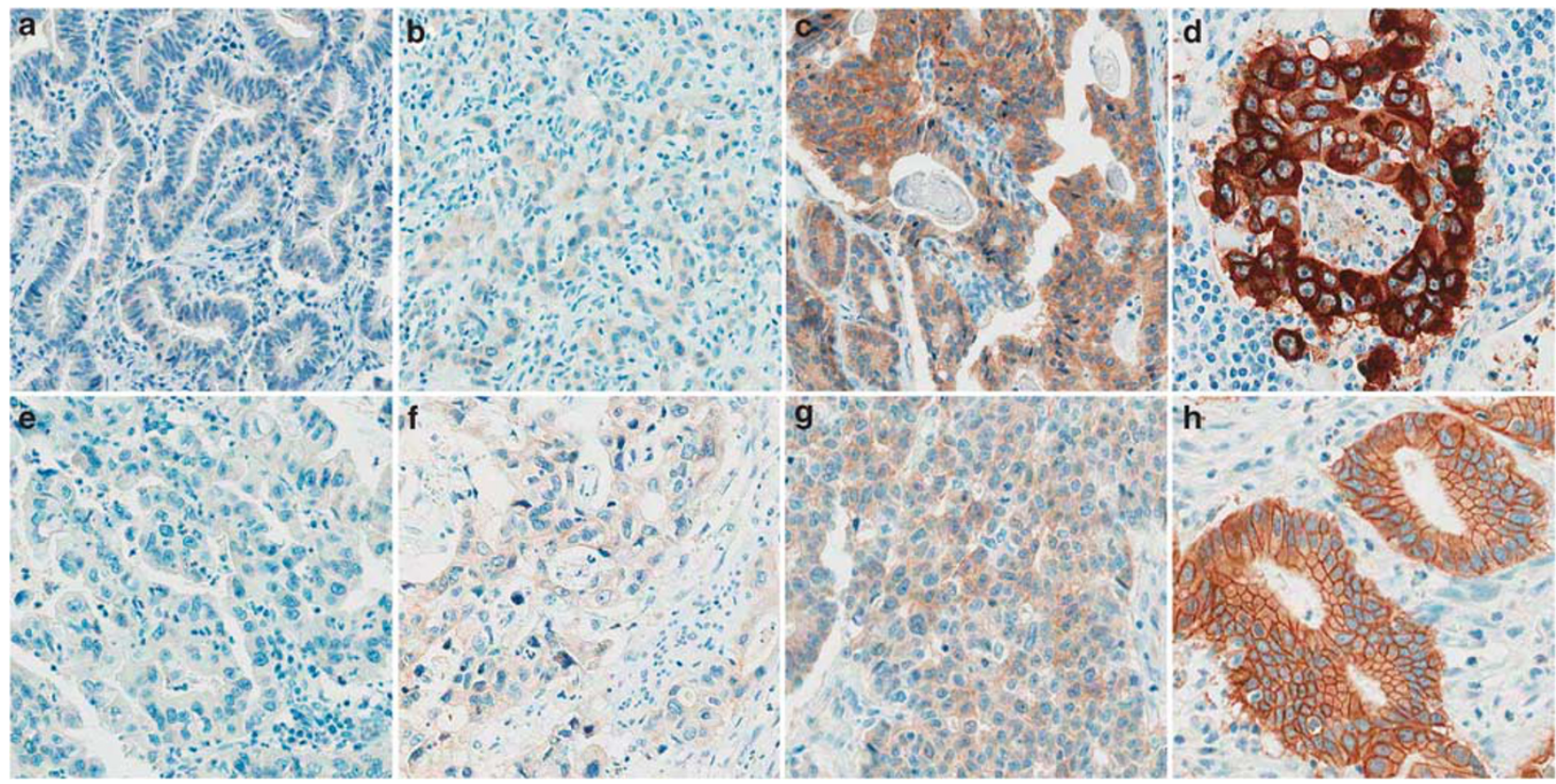

Figure 1 Representative photomicrographs of MET expression interpreted as $0(\mathbf{a}), 1+(\mathbf{b}), 2+(\mathbf{c})$ and $3+(\mathbf{d})$ in both membrane and cytoplasm. MET expression scored as $0(\mathbf{e}), 1+(\mathbf{f}), 2+(\mathbf{g})$ and $3+(\mathbf{h})$ in the membrane of tumor cells.

\section{MET Copy Number Gain by Real-Time Quantitative PCR}

Genomic DNAs from 120 randomly selected, formalin-fixed, paraffin-embedded gastric carcinoma tumor tissues were extracted with a gDNA extraction kit (Qiagen, Valencia, CA, USA). The reaction mixture contained $2 \mu \mathrm{l}$ genomic DNA template, $10 \mu \mathrm{l}$ of Taqman universal PCR master mixture (Applied Biosystems, Foster City, CA, USA) and $0.2 \mu \mathrm{M}$ of each primer. To accurately detect CNG, we analyzed three different regions of the MET gene: a region spanning exon 3-intron 3 boundaries (TaqMan Copy Number Assay Hs01602615_cn), a region within intron 12 (Hs0527935_cn) and a region spanning intron 20-exon 21 boundaries (Hs02884964_cn). ${ }^{3}$ Moreover, previously described probes by Smolen et $a l^{15}$ were also added for analyses. The region of the probes in MET gene is depicted in Figure 2.

Copy number gain was measured with the following profile: $2 \mathrm{~min}$ at $50{ }^{\circ} \mathrm{C}$, denaturation at $95{ }^{\circ} \mathrm{C}$ for $10 \mathrm{~min}$, followed by 40 cycles of $95^{\circ} \mathrm{C}$ for $15 \mathrm{~s}$ and $60^{\circ} \mathrm{C}$ for $1 \mathrm{~min}$ using relative quantification using 7900 HT fast real-time PCR system in quadruplicate. A RNaseP assay kit (Applied Biosystems) was used as a control. After amplification, the experiment results containing threshold cycle (Ct) values for the copy number and reference assay was imported into the CopyCaller Software (Applied Biosystems) for post-PCR data analysis as previously described. ${ }^{3}$ The copy number gain status and the number of MET copies were assigned on the basis of concordance of result in at least two of the four MET regions of probe.

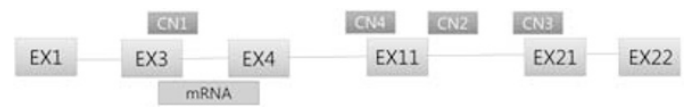

Figure 2 A schematic diagram showing four probe regions for copy number gain (CN1, CN2, CN3 and CN4) and mRNA levels of $M E T$ gene used for real-time quantitative PCR.

\section{Quantification of MET mRNA Using Quantitative Real-Time PCR}

Total RNA extractions were performed from the same 120 formalin-fixed, paraffin-embedded gastric carcinomas used for copy number gain with an RNeasy FFPE kit (Qiagen). Total RNA concentration was measured by fluorescence using a Quant-iT RNA Assay Kit (Invitrogen, Eugene, OR, USA). RNAs were reverse transcribed using a High Capacity cDNA Reverse Transcription Kit (Applied Biosystems). Quantitative real-time PCR was carried out with using specific TaqMan Gene Expression Assays (Applied Biosystems assay ID; Hs01565584_m1). GAPDH gene (ID; Hs99999905 _m1) was used as an endogenous control for RNA quality. Two independent RT reactions were performed for all samples. The comparative Ct $(\Delta \Delta \mathrm{Ct})$ method calculated the difference $(\Delta \mathrm{Ct})$ between the threshold cycles of the target and reference genes.

\section{Fluorescent and Bright-Field Double In Situ Hybridization}

In 25 selected cases including all 9 membranous and cytoplasmic MET $3+$ cases, FISH was performed using dual-color DNA-specific MET/CEP7 probes 
(Abnova, Walnut, CA, USA) as described previously. $^{31}$ For bright-field double in situ hybridization, the INFORM MET DNA probe was used in the same block using a Ventana ultraViewTM SISH Detection Kit on a Ventana BenchMark XT automated slide stainer following the instructions provided by the manufacturer (Ventana Medical Systems). Three pathologists (SYH, IGD and KMK) counted the numbers of MET and chromosome 7 centromere probe (CEP7) signals (1 for individual signals, 6 for small clusters and 12 for big clusters) in 20 inter-phase tumor cell nuclei, and the mean number of MET and CEP7 copies per nucleus were determined, along with the ratio. Normal MET/CEP7 signals (one to two copies per cell) in the various non-neoplastic cells served as the internal positive control. MET amplification followed strict definitions as established for HER2 testing. ${ }^{32}$ Briefly, we defined gene amplification as a MET/CEP7 ratio > 2.0 in 20 tumor nuclei. Low or high polysomy were regarded as negative for gene amplification.

\section{Statistical Analysis}

For analysis of the relationship between clinicopathologic characteristics and MET overexpression, Pearson's $\chi^{2}$ test was used. The Cochran-Armitage trend test was used to see if there existed an increasing trend of MET immune-positivity according to $\mathrm{N}$ stage and MET copy number gain. One-way analysis of variance was used to compare mean value of mRNA expression level in different groups of immunohistochemistry scores. The Pearson correlation test was used to evaluate the correlation between mRNA level and immunohistochemistry score. Disease-free and overall survivals were determined using the Kaplan-Meier method, and survival curves were compared using the log-ratio method. Survival was measured from the date of surgery. The Cox proportional hazard model was used to evaluate the associations between clinicopathologic factors and survival. The hazard ratio and its 95\% confidence interval were assessed for each factor. All tests were two sided, and $P$-values $<0.05$ were considered to be statistically significant. Statistical analysis was performed using SPSS software (SPSS., Chicago, IL, USA) and R software (version 2.12.0 for windows).

\section{Results}

\section{Overexpression of MET and Clinicopathologic Characteristics}

Simultaneous membranous and cytoplasmic MET expression was scored as 0 in 167 (34\%), $1+$ in 220 $(44 \%), 2+$ in $99(20 \%)$ and $3+$ in $9(2 \%)$ gastric cancers and membranous and cytoplasmic overexpression of MET $(2+$ and $3+)$ was found in 108 cases $(22 \%)$. Clinicopathologic findings observed in the gastric carcinomas with or without MET overexpression are summarized in Table 1. KaplanMeier survival analyses showed that patients with overexpression of MET showed significantly shorter overall $(P=0.002)$ and disease-free $(P=0.004)$ survivals compared with patients without overexpression (Figure 3). To confirm MET membranous and cytoplasmic overexpression as independent prognostic factors, we performed multivariate Cox proportional hazard model analyses, which included age, gender, differentiation, Lauren classification, $\mathrm{pN}$ stage, distant metastasis and MET expression status as covariates. In addition to $\mathrm{pN}$ stage and the presence of distant metastasis, membranous and cytoplasmic overexpression of MET remained significant in both overall survival (HR, 1.781; 95\% CI, 1.324-2.395; $P<0.001$ ) and disease-free survival (HR, 1.765; 95\% CI, 1.2272.541; $P=0.002$; Table 2).

Of 495 patients, membranous MET expression was scored as 0 in $199(40 \%), 1+$ in $189(38 \%), 2+$ in $67(14 \%)$ and $3+$ in $40(8 \%)$ gastric cancers and membranous overexpression of MET $(3+)$ was found in 40 cases (8\%). Clinicopathologic findings observed in the gastric carcinomas with or without MET membranous overexpression are summarized in Table 1. Membranous MET overexpression was significantly associated with male gender $(P=0.049)$, intestinal type by Lauren and differentiated histology $(P=0.001)$. However, it was not associated with overall survival $(P=0.076)$ or disease-free survival $(P=0.131$; Figure 3$)$.

\section{mRNA Expression, Copy Number Gain and MET Immunohistochemistry}

mRNA expression levels correlated more strongly with immunohistochemistry interpreted by membranous and cytoplasmic staining than membranous alone staining. MET mRNA expression levels showed a moderately positive correlation with MET membranous and cytoplasmic staining (Pearson correlation coefficient, $r=0.491, P<0.001$ ), but a weakly positive correlation with membranous staining alone $(r=0.274, P=0.002$; Figure 4$)$.

$M E T$ copy number gains with $>4$ copies were found in 14 out of 120 examined cases $(12 \%)$. Seven out of $8(88 \%)$ MET membranous and cytoplasmic $3+$ cases and 7 out of 49 (14\%) MET membranous and cytoplasmic $2+$ gastric carcinomas showed copy number gains, while only $33 \%$ of MET membranous 3+ cases and 7\% of MET membranous $2+$ cases did. None of the MET membranous and cytoplasmic 0 or $1+$ cancers demonstrated MET copy number gain (Table 3 ).

\section{MET FISH and Dual Bright-Field In Situ Hybridization}

The results of FISH correlated perfectly with dual bright-field in situ hybridization. The results of 
Table 1 Patient characteristics according to MET protein overexpression in the cytoplasm or membrane of tumor cells

\begin{tabular}{|c|c|c|c|c|c|c|}
\hline \multirow[b]{2}{*}{ Characteristics, n (\%) } & \multicolumn{3}{|c|}{ MET membranous and cytoplasmic staining } & \multicolumn{3}{|c|}{ MET membranous staining } \\
\hline & Positive $(\mathrm{n}=108)$ & Negative $(\mathrm{n}=387)$ & P-value & Positive $(\mathrm{n}=40)$ & Negative $(\mathrm{n}=455)$ & $\mathrm{P}$-value \\
\hline \multicolumn{7}{|l|}{ Gender } \\
\hline Male & $74(69)$ & $252(65)$ & 0.734 & $32(80)$ & $294(65)$ & 0.049 \\
\hline Female & $34(32)$ & $135(35)$ & & $8(20)$ & $161(35)$ & \\
\hline \multicolumn{7}{|l|}{ Age (years) } \\
\hline$<60$ & $60(56)$ & $249(64)$ & 0.088 & $22(55)$ & $287(63)$ & 0.312 \\
\hline$\geq 60$ & $48(44)$ & $138(36)$ & & $18(45)$ & $168(17)$ & \\
\hline \multicolumn{7}{|l|}{ Tumor location } \\
\hline Upper third & $16(15)$ & $63(16)$ & 0.854 & $6(15)$ & $73(16)$ & 0.444 \\
\hline Middle third & $42(39)$ & $158(41)$ & & $12(30)$ & $188(41)$ & \\
\hline Lower third & $43(40)$ & $140(36)$ & & $18(45)$ & $165(36)$ & \\
\hline Whole stomach & $7(7)$ & $26(7)$ & & $4(10)$ & $29(6)$ & \\
\hline \multicolumn{7}{|l|}{ Tumor size } \\
\hline$<4.5$ & $27(25)$ & $85(22)$ & 0.504 & $8(20)$ & $104(23)$ & 0.679 \\
\hline$\geq 4.5$ & $81(75)$ & $302(78)$ & & $32(80)$ & $351(77)$ & \\
\hline \multicolumn{7}{|l|}{ Lauren classification } \\
\hline Diffuse & $50(46)$ & $243(63)$ & 0.015 & $13(33)$ & $280(62)$ & 0.001 \\
\hline Intestinal & $53(49)$ & $121(31)$ & & $25(63)$ & 149 (33) & \\
\hline Mixed & $5(5)$ & $23(6)$ & & $2(5)$ & $26(6)$ & \\
\hline \multicolumn{7}{|l|}{ Histology } \\
\hline Differentiated & $44(41)$ & $120(31)$ & 0.15 & $23(58)$ & $141(31)$ & 0.001 \\
\hline Undifferentiated & $64(59)$ & $267(69)$ & & $17(43)$ & 314 (69) & \\
\hline \multicolumn{7}{|l|}{ Lymphatic invasion } \\
\hline Negative & $49(45)$ & $200(52)$ & 0.18 & $18(45)$ & $231(51)$ & 0.484 \\
\hline Positive & $59(55)$ & $187(48)$ & & $22(55)$ & $224(49)$ & \\
\hline \multicolumn{7}{|l|}{ Vacular invasion } \\
\hline Negative & $99(92)$ & $342(88)$ & 0.648 & $36(90)$ & 405 (89) & 0.847 \\
\hline Positive & $9(8)$ & $45(12)$ & & $4(10)$ & $50(11)$ & \\
\hline \multicolumn{7}{|l|}{ Perineural invasion } \\
\hline Negative & $76(70)$ & 267 (69) & 0.575 & $31(78)$ & 312 (69) & 0.241 \\
\hline Positive & $32(30)$ & $120(31)$ & & $9(23)$ & $143(31)$ & \\
\hline \multicolumn{7}{|l|}{$N$ stage } \\
\hline No & $16(15)$ & $79(20)$ & $0.448^{\mathrm{a}}$ & $6(15)$ & $89(20)$ & $0.133^{\mathrm{a}}$ \\
\hline N1 & $20(19)$ & $76(20)$ & & 6 (15) & $90(20)$ & \\
\hline N2 & $24(22)$ & $71(18)$ & & $8(20)$ & 87 (19) & \\
\hline N3a & $22(20)$ & $94(24)$ & & $8(20)$ & $108(24)$ & \\
\hline N3b & $26(24)$ & 67 (17) & & $12(30)$ & 81 (18) & \\
\hline \multicolumn{7}{|l|}{ Metastasis } \\
\hline Negative & $105(97)$ & $380(98)$ & 0.529 & $38(95)$ & 447 (98) & 0.162 \\
\hline Positive & $3(3)$ & $7(2)$ & & $2(5)$ & $8(2)$ & \\
\hline \multicolumn{7}{|l|}{ Recurrence of disease } \\
\hline Yes & $44(41)$ & $118(31)$ & 0.045 & $15(38)$ & $147(32)$ & 0.502 \\
\hline No & $64(59)$ & $269(70)$ & & $25(63)$ & $308(68)$ & \\
\hline \multicolumn{7}{|l|}{ Died of disease } \\
\hline Yes & $64(59)$ & $181(47)$ & 0.022 & $23(58)$ & $222(49)$ & 0.291 \\
\hline No & $44(41)$ & $206(53)$ & & $17(43)$ & $233(51)$ & \\
\hline
\end{tabular}

a By Cochran-Armitage trend test, otherwise by Pearson $\chi^{2}$ test.

in situ hybridization and corresponding immunohistochemistry, copy number gain and mRNA expression levels in selected cases are summarized in Table 4. Increased mRNA levels and copy number gains were observed in all four examined membranous and cytoplasmic $3+$ cases. Moreover, all nine membranous and cytoplasmic MET $3+$ cases showed amplification of MET gene with several big 
a

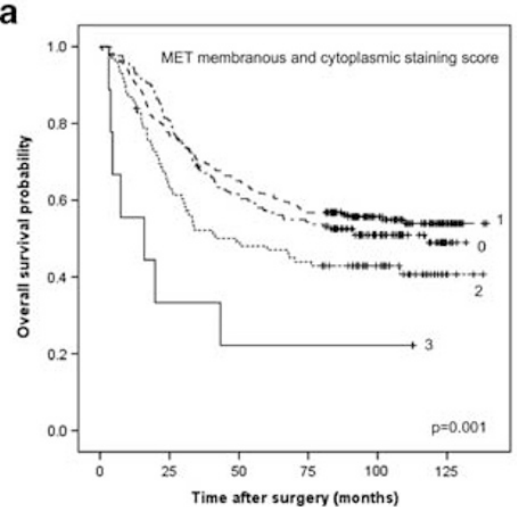

d

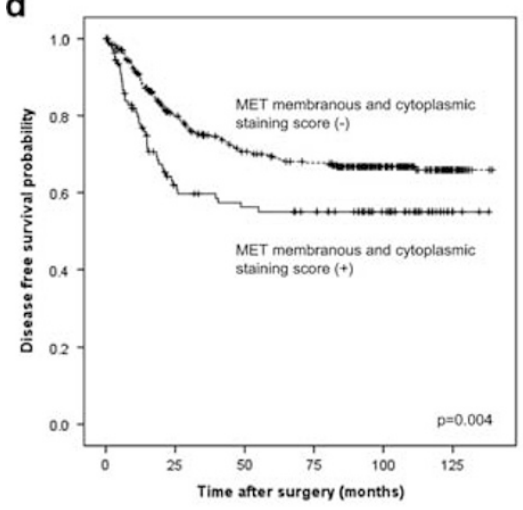

b

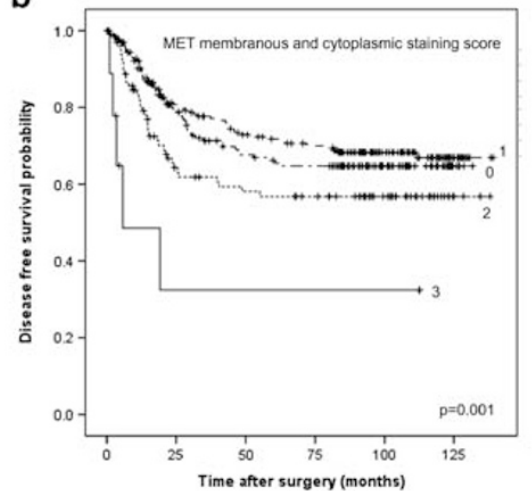

e

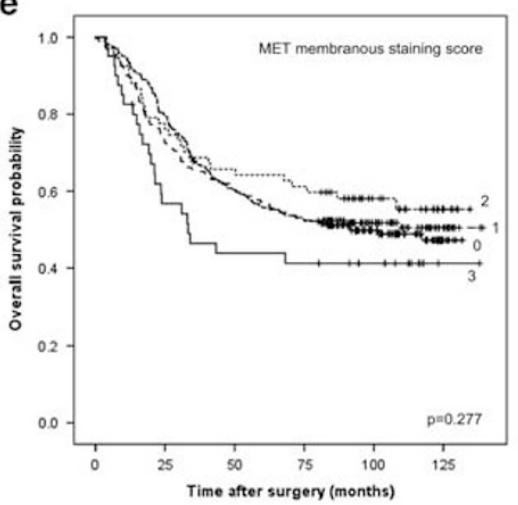

C

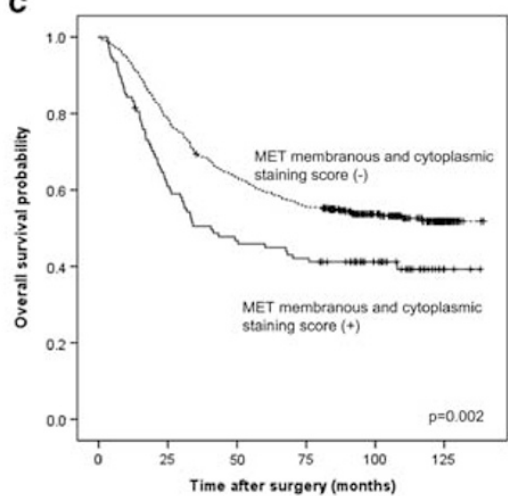

f

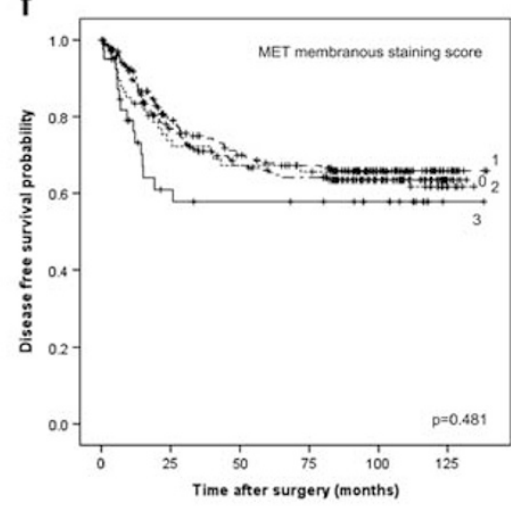

g

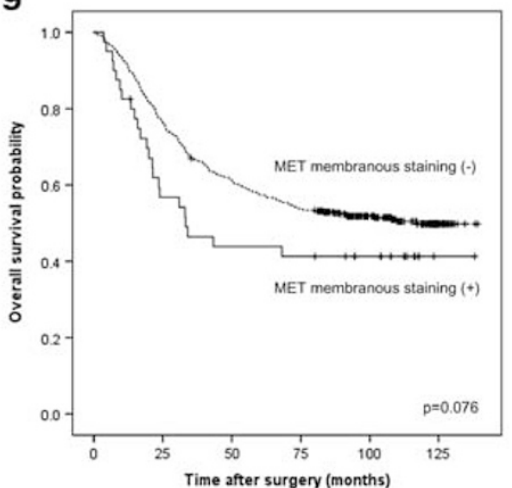

h

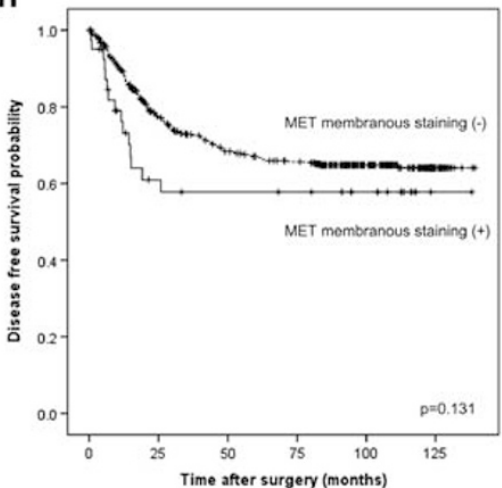

Figure 3 (a) Overall survival and (b) disease-free survival curves of 495 patients according to the scores of membranous and cytoplasmic staining of MET immunohistochemistry. (c) Overall survival and (d) disease-free survival curves reveal poorer prognosis in patients with MET membranous and cytoplasmic overexpression compared with negative group. (e) Overall survival and (f) disease-free survival curves of 495 patients according to the scores of membranous staining of MET by immunohistochemistry. (g) Overall survival and (h) disease-free survival curves reveal poorer prognosis in MET membranous overexpression group, but did not reach statistical significance.

clusters of signal in the nuclei (Figures 5a-i). Similarly, the MET-amplified positive controls, MKN45 and SNU5 GC cell lines showed many large clustered signals of MET gene in the tumor cell nuclei (Figures 5j-l). However, membranous and cytoplasmic MET $2+(n=12)$ or $0 \quad(n=4)$ cases showed no amplification of MET gene although mRNA levels were slightly increased. Six membranous $3+$ cases also showed no amplification of $M E T$ gene.

\section{Discussion}

To better characterize MET-positive gastric cancers, immunohistochemistry for MET protein was performed in 495 advanced gastric carcinomas. We defined new interpretation methods of MET overexpression in gastric carcinoma. Membranous and cytoplasmic MET overexpression was observed in 108 cases (22\%), which correlated well with increased copy number gain, mRNA levels and 
Table 2 Multivariate Cox proportional hazard model analyses by MET immunohistochemistry

\begin{tabular}{|c|c|c|c|c|c|c|}
\hline \multirow[b]{2}{*}{ Multivariate Cox proportional hazard model analysis } & \multicolumn{3}{|c|}{ Overall survival } & \multicolumn{3}{|c|}{ Disease-free survival } \\
\hline & Hazard ratio & $C I$ & $\mathrm{P}$-value & Hazard ratio & $C I$ & $\mathrm{P}$-value \\
\hline MET expression, negative vs overexpression & 1.781 & $1.324-2.395$ & $<0.001$ & 1.765 & $1.227-2.541$ & 0.002 \\
\hline Lauren classification, intestinal vs diffuse or mixed & 1.301 & $0.834-2.030$ & 0.246 & 1.695 & $0.949-3.029$ & 0.075 \\
\hline Metastasis, absent vs present & 2.843 & $1.444-5.596$ & 0.003 & 2.411 & $0.979-5.940$ & 0.056 \\
\hline pN stage, $\mathrm{N} 0,1$ vs $\mathrm{N} 2,3$ & 2.379 & $1.781-3.179$ & $<0.001$ & 1.888 & $1.340-2.661$ & $<0.001$ \\
\hline Age, $<60$ years $v s \geq 60$ years & 1.564 & $1.208-2.026$ & 0.001 & 1.380 & $1.001-1.903$ & 0.049 \\
\hline Gender, female vs male & 1.092 & $0.836-1.426$ & 0.520 & 1.103 & $0.796-1.526$ & 0.556 \\
\hline Differentiation, differentiated vs undifferentiated & 0.954 & $0.609-1.496$ & 0.838 & 1.072 & $0.599-1.918$ & 0.814 \\
\hline
\end{tabular}
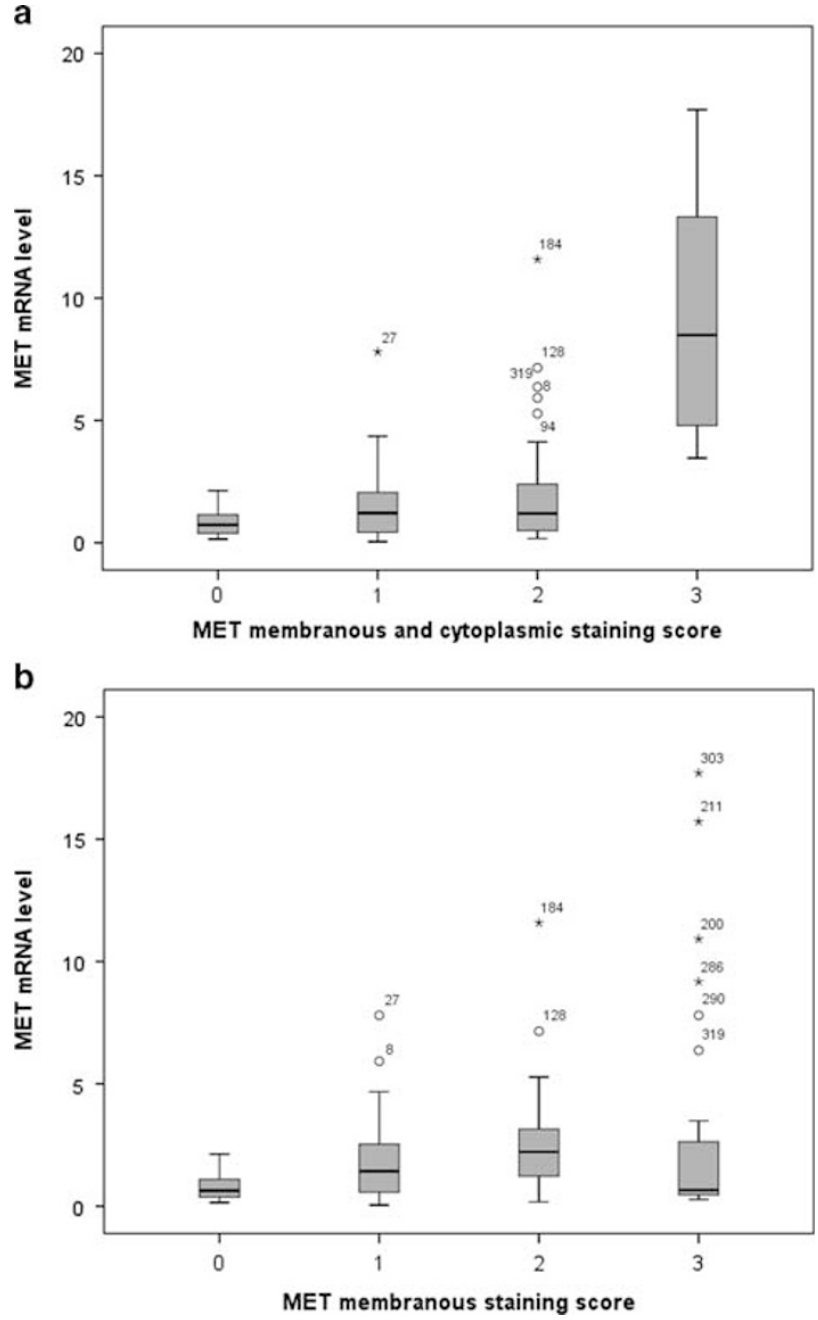

Figure 4 (a) mRNA levels of MET gene relative to GAPDH in each score of both membranous and cytoplasmic staining. (b) mRNA levels of MET gene relative to GAPDH in each score of membranous staining. The bar within each graph indicates the median value.

MET gene amplification, and was an independent prognosticator of poor survival in gastric carcinomas, supporting the clinical impact of MET protooncogene activation in gastric cancers. Moreover, all nine membranous and cytoplasmic MET $3+$ cases showed amplification of MET gene.
In previous studies on gastric MET, the positivity rate in protein expression by immunohistochemistry (24 to $74 \%$ ), gene amplification (0-23\%) and copy number gain (9-23\%) were highly variable. ${ }^{5,7,11,14,16-23,33}$ Although MET expression showed poor prognostic effects on gastric carcinomas, ${ }^{7,18,20,21,23}$ most studies were performed $>10$ years $\mathrm{ago}^{5,7,17,19-22}$ and interpreted either cytoplasmic or membranous positivity only,17-21 without considering intensity. In a recent large cohort study with gastric cancers with the same MET monoclonal antibody as ours, interpretation of membranous staining, which was used for HER2, failed to find any clinical significance and not all MET $3+$ cases exhibited amplification of MET gene. ${ }^{16}$ The lack of a reliable method for evaluating MET immunohistochemistry has caused discrepancies in previous studies. In our comprehensive study, we first proved that both membranous and cytoplasmic MET staining correlate well with mRNA expression levels, amplification of MET gene and clinical outcome. Moreover, we investigated copy number gain with two methods: real-time quantitative PCR and in situ hybridization methods. In PCR, copy number gains with $>3$ copies were observed with variable frequency, and copy number gains of $>5$ copies were mainly observed in immunohistochemistry $3+$ cases. However, considerable numbers of $2+$ positive cases showed no copy number gains. Recently, Graziano et $a l^{3}$ investigated $M E T$ copy number gain by PCR in 216 gastric carcinomas, and $10 \%$ of patients with copy number gains showed poorer prognosis. In that study, true amplification of MET gene was not observed in FISH. Janjigian et $a l^{24}$ also showed that true amplification of MET in gastric cancer is absent. However, earlier reports from Japan showed amplification by FISH in $3.9 \%$ of advanced gastric carcinomas. ${ }^{6}$ Recent studies from the United States and Korea showed true MET amplification in $2 \%$ of gastroesophageal ${ }^{23}$ and gastric ${ }^{20}$ adenocarcinomas. Similarly, we also detected amplification of MET gene in nine cases $(2 \%)$, where MET immunohistochemistry was $3+$ stained in the cytoplasm and membrane of tumor cells.

Given the low incidence of MET gene amplification, it is not feasible in the clinic to screen every gastric cancer patient by MET FISH. We extensively 
Table 3 Correlation between MET immunohistochemistry and MET copy number

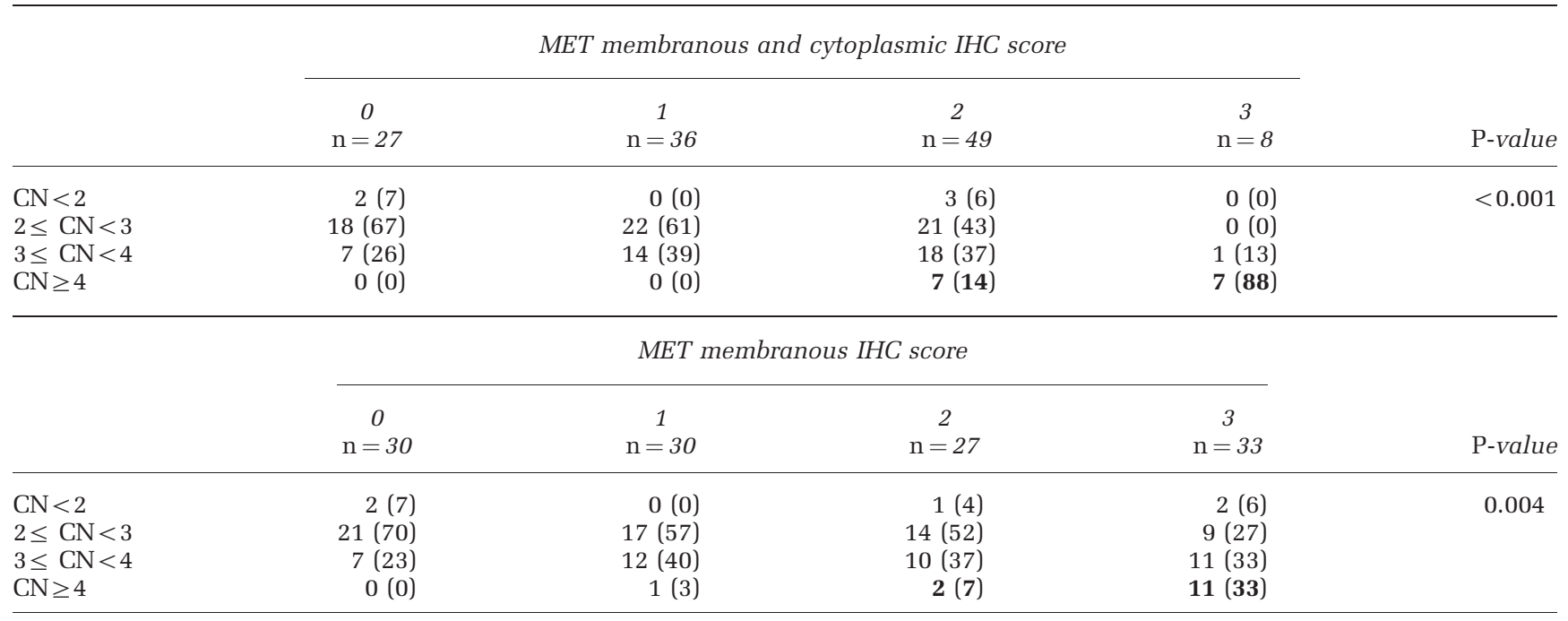

Abbreviations: CN, copy number; IHC, immunohistochemistry.

Bold entries indicate cases with copy gain number $\geqslant 4$ by Fisher's exact test.

Table 4 Results of dual silver in situ hybridization, fluorescent in situ hybridization, immunohistochemistry, copy number gain and mRNA levels of MET gene in 20 selected cases and cell lines

\begin{tabular}{|c|c|c|c|c|c|c|c|c|c|c|c|c|c|}
\hline \multirow[b]{2}{*}{ Cases } & \multicolumn{3}{|c|}{$\begin{array}{l}\text { Dual in situ } \\
\text { hybridization }\end{array}$} & \multicolumn{3}{|c|}{$\begin{array}{l}\text { Fluorescent in situ } \\
\text { hybridization }\end{array}$} & \multirow[b]{2}{*}{$\begin{array}{l}\text { Membranous and } \\
\text { Cytoplasmic MET } \\
\text { scores }\end{array}$} & \multirow[b]{2}{*}{$\begin{array}{c}\text { Membranous } \\
\text { MET scores }\end{array}$} & \multirow{2}{*}{\multicolumn{2}{|c|}{ CN1 CN2 }} & \multirow[b]{2}{*}{ CN3 } & \multirow{2}{*}{\multicolumn{2}{|c|}{$\begin{array}{c}m R N \\
A \\
\text { CN4 levels }\end{array}$}} \\
\hline & $\begin{array}{c}\text { Ratio of } \\
\text { MET/ } \\
\text { CEP7 }\end{array}$ & $\begin{array}{c}\text { Copy } \\
\text { number of } \\
\text { MET }\end{array}$ & $\begin{array}{c}\text { Copy } \\
\text { number of } \\
\text { CEP7 }\end{array}$ & $\begin{array}{c}\text { Ratio of } \\
\text { MET/ } \\
\text { CEP7 }\end{array}$ & $\begin{array}{c}\text { Copy } \\
\text { number o } \\
\text { MET }\end{array}$ & $\begin{array}{c}\text { Copy } \\
\text { f number of } \\
\text { CEP7 }\end{array}$ & & & & & & & \\
\hline 1 & 1.17 & 2.52 & 2.16 & 0.97 & 2.24 & 2.32 & 2 & 2 & 2 & 3 & 3 & 2 & 0.272 \\
\hline 2 & 1.06 & 2.04 & 1.92 & 1.06 & 2.08 & 1.96 & 2 & 3 & 2 & 1 & 2 & 1 & 2.635 \\
\hline 3 & 1.06 & 2.64 & 2.48 & 0.95 & 2.32 & 2.44 & 2 & 3 & 2 & 3 & 3 & 2 & 0.657 \\
\hline 4 & 1.13 & 2.44 & 2.16 & 0.96 & 2.56 & 2.68 & 2 & 3 & 3 & 2 & 2 & 2 & 0.394 \\
\hline 5 & 1.00 & 2.2 & 2.2 & 0.98 & 2.08 & 2.12 & 0 & 1 & 2 & 1 & 1 & 1 & 0.247 \\
\hline 6 & 0.87 & 2.08 & 2.40 & 0.81 & 1.84 & 2.28 & 2 & 3 & 2 & 4 & 5 & 2 & 1.361 \\
\hline 7 & 1.12 & 2.2 & 1.96 & 0.87 & 1.88 & 2.16 & 0 & 1 & 2 & 2 & 3 & 1 & 0.150 \\
\hline 8 & 1.17 & 2.72 & 2.32 & 1.02 & 2.08 & 2.04 & 2 & 2 & 2 & 2 & 2 & 2 & 0.701 \\
\hline 9 & 8.97 & 22.60 & 2.52 & 8.50 & 20.40 & 2.40 & 3 & 3 & 3 & 4 & 1 & 4 & 3.458 \\
\hline 10 & 10.96 & 24.56 & 2.24 & 9.96 & 22.72 & 2.28 & 3 & 3 & 10 & 6 & 8 & 8 & 15.714 \\
\hline 11 & 1.00 & 2.44 & 2.44 & 1.04 & 2.16 & 2.08 & 2 & 0 & 2 & 1 & 1 & 2 & 0.544 \\
\hline 12 & 1.05 & 2.60 & 2.48 & 0.91 & 2.48 & 2.72 & 2 & 2 & 2 & 4 & 4 & 2 & 0.476 \\
\hline 13 & 1.06 & 2.16 & 2.04 & 0.96 & 2.00 & 2.08 & 2 & 3 & 2 & 2 & 2 & 2 & 0.331 \\
\hline 14 & 12.27 & 31.40 & 2.56 & 10.91 & 25.32 & 2.32 & 3 & 3 & 8 & 3 & 2 & 9 & 9.177 \\
\hline 15 & 1.02 & 2.16 & 2.12 & 1.10 & 2.16 & 1.96 & 2 & 0 & 3 & 1 & 1 & 2 & 0.631 \\
\hline 16 & 9.59 & 22.24 & 2.32 & 7.41 & 15.12 & 2.04 & 3 & 3 & 3 & 4 & 4 & 3 & 4.678 \\
\hline 17 & 1.02 & 2.08 & 2.04 & 0.98 & 2.00 & 2.04 & 0 & 2 & 2 & 1 & 1 & 2 & 1.094 \\
\hline 18 & 1.13 & 2.16 & 1.92 & 0.94 & 2.00 & 2.12 & 0 & 2 & 2 & 3 & 4 & 2 & 0.944 \\
\hline 19 & 1.09 & 2.48 & 2.28 & 0.97 & 2.40 & 2.48 & 2 & 3 & 2 & 3 & 3 & 2 & 0.412 \\
\hline 20 & 1.02 & 2.20 & 2.16 & 1.04 & 2.20 & 2.12 & 2 & 1 & 2 & 1 & 1 & 1 & 0.503 \\
\hline MKN45 & 12.16 & 24.80 & 2.04 & 8.13 & 14.64 & 1.90 & 3 & 3 & 26 & 23 & 23 & 20 & 2.469 \\
\hline SNU5 & 8.25 & 19.80 & 2.40 & 7.07 & 15.28 & 2.16 & 3 & 3 & 22 & 21 & 21 & 16 & 6.699 \\
\hline SNU1 & 1.16 & 2.26 & 1.95 & 1.15 & 2.12 & 1.84 & 0 & 0 & 2 & 3 & 2 & 2 & 0 \\
\hline
\end{tabular}

Abbreviation: CN, copy number.

Bold entries indicate cases with amplification of MET gene by in situ hybridization.

analyzed the association between MET protein overexpression, MET mRNA expression and MET gene copy number gain using all four known probes and MET amplification by in situ hybridization. Given the major advantage of immunohistochemistry, routine applicability in the clinic and cost 

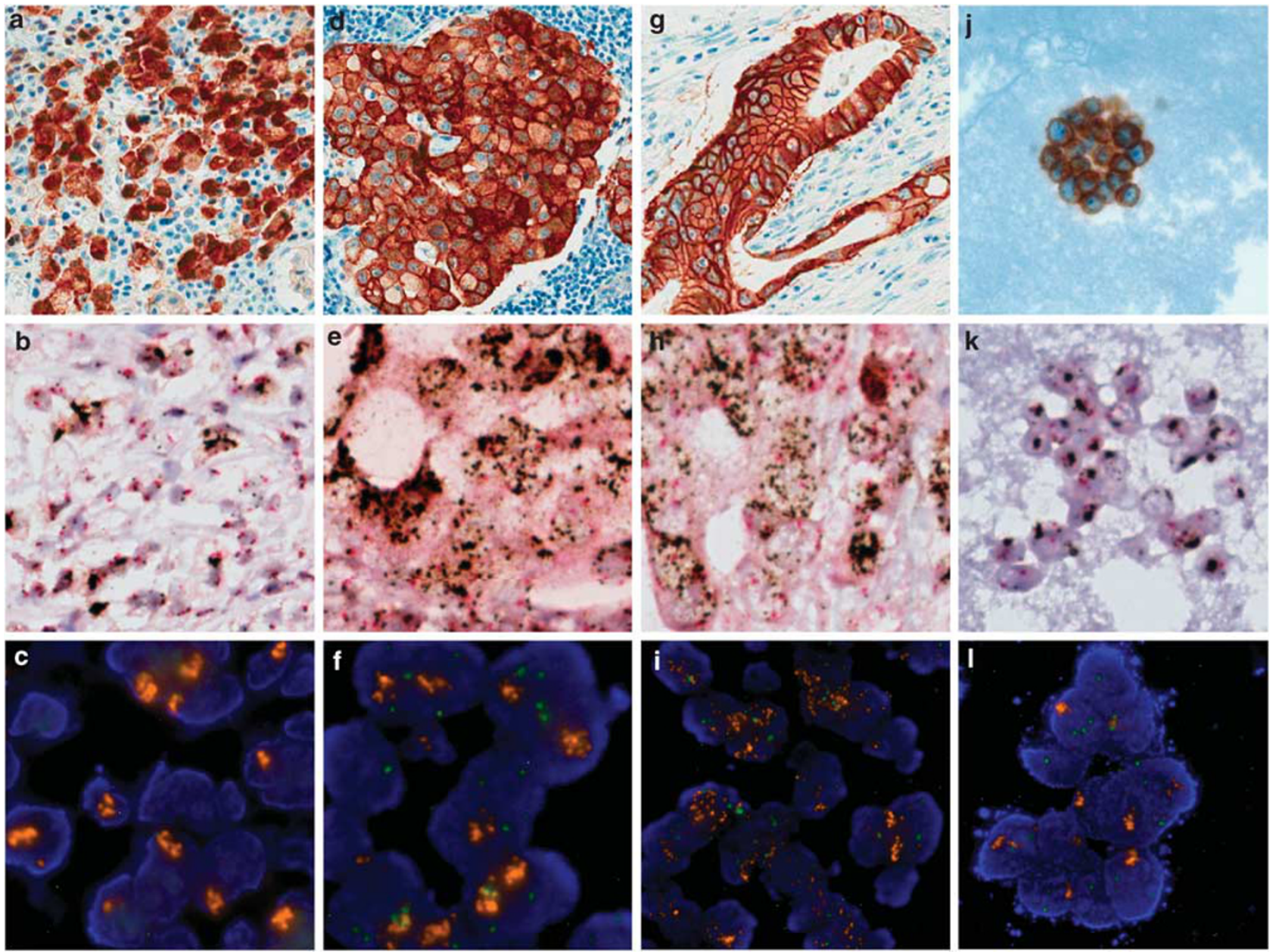

Figure 5 Photomicrograph of three representative cases (a-c, $\mathbf{d}-\mathbf{f}, \mathbf{g}-\mathbf{i})$ and one cell line (MKN45: j-l) with amplification of MET gene; matched sets of immunohistochemical staining results (upper row) with dual in situ hybridization (middle row) and fluorescence in situ hybridization (lower row).

effectiveness, our results strongly recommend MET immunohistochemistry as an initial screening test for MET amplification. MET inhibitor therapy would be indicated for patients with membranous and cytoplasmic MET $3+$ or MET-amplified gastric cancer. In MET $2+$ gastric cancers, although gene amplification was not observed in our limited cases, further confirmation should be performed using either fluorescent or silver in situ hybridizations. However, as patients with membranous and cytoplasmic MET $2+$ showed similarly poor survival as those with MET $3+$, some (14\%) MET $2+$ gastric carcinomas showed copy number gains, and MET protein overexpression could not be explained fully by gene amplification, ${ }^{3,24}$ we defined MET $2+$ cases as overexpression.

In conclusion, interpretation of MET immunohistochemistry based on membranous and cytoplasmic staining correlated very well with mRNA expression, copy number gain and amplification of MET gene. In gastric cancers, MET overexpression is an independent prognostic factor and could be a good potential target and predictor of benefit for MET inhibitor therapy.

\section{Acknowledgements}

This study was supported by a grant from the Korea Healthcare Technology R\&D Project, Ministry for Health and Welfare Affairs, Republic of Korea (A092255 and A101130) and a Samsung Biomedical Research Institute grant (\#SBRI-CB11031).

\section{Disclosure/conflict of interest}

The authors declare no conflict of interest.

\section{References}

1 Eder JP, Vande Woude GF, Boerner SA, et al. Novel therapeutic inhibitors of the c-Met signaling pathway in cancer. Clin Cancer Res 2009;15:2207-2214.

2 Bang YJ, Van Cutsem E, Feyereislova A, et al. Trastuzumab in combination with chemotherapy versus chemotherapy alone for treatment of HER2-positive advanced gastric or gastro-oesophageal junction cancer (ToGA): a phase 3, open-label, randomised controlled trial. Lancet 2010;376:687-697. 
3 Graziano F, Galluccio N, Lorenzini P, et al. Genetic activation of the MET pathway and prognosis of patients with high-risk, radically resected gastric cancer. J Clin Oncol 2011;29:4789-4795.

4 Yap TA, Olmos D, Brunetto AT, et al. Phase I trial of a selective c-MET inhibitor ARQ 197 incorporating proof of mechanism pharmacodynamic studies. J Clin Oncol 2011;29:1271-1279.

5 Park WS, Oh RR, Kim YS, et al. Absence of mutations in the kinase domain of the Met gene and frequent expression of Met and HGF/SF protein in primary gastric carcinomas. APMIS 2000;108:195-200.

6 Hara T, Ooi A, Kobayashi M, et al. Amplification of c-myc, K-sam, and c-met in gastric cancers: detection by fluorescence in situ hybridization. Lab Invest 1998;78:1143-1153.

7 Nakajima M, Sawada H, Yamada Y, et al. The prognostic significance of amplification and overexpression of c-met and c-erb B-2 in human gastric carcinomas. Cancer 1999;85:1894-1902.

8 Tsugawa K, Yonemura Y, Hirono Y, et al. Amplification of the c-met, c-erbB-2 and epidermal growth factor receptor gene in human gastric cancers: correlation to clinical features. Oncology 1998;55:475-481.

9 Seruca R, Suijkerbuijk RF, Gartner F, et al. Increasing levels of MYC and MET co-amplification during tumor progression of a case of gastric cancer. Cancer Genet Cytogenet 1995;82:140-145.

10 Nessling M, Solinas-Toldo S, Wilgenbus KK, et al. Mapping of chromosomal imbalances in gastric adenocarcinoma revealed amplified protooncogenes MYCN, MET, WNT2, and ERBB2. Genes Chromosomes Cancer 1998;23:307-316.

11 Kuniyasu H, Yasui W, Kitadai Y, et al. Frequent amplification of the c-met gene in scirrhous type stomach cancer. Biochem Biophys Res Commun 1992;189:227-232.

12 Tsujimoto H, Sugihara H, Hagiwara A, et al. Amplification of growth factor receptor genes and DNA ploidy pattern in the progression of gastric cancer. Virchows Arch 1997;431:383-389.

13 Sakakura C, Mori T, Sakabe T, et al. Gains, losses, and amplifications of genomic materials in primary gastric cancers analyzed by comparative genomic hybridization. Genes Chromosomes Cancer 1999;24: 299-305.

14 Lee J, Seo JW, Jun HJ, et al. Impact of MET amplification on gastric cancer: possible roles as a novel prognostic marker and a potential therapeutic target. Oncol Rep 2011;25:1517-1524.

15 Smolen GA, Sordella R, Muir B, et al. Amplification of MET may identify a subset of cancers with extreme sensitivity to the selective tyrosine kinase inhibitor PHA-665752. Proc Natl Acad Sci USA 2006;103: 2316-2321.

16 Lee HE, Kim MA, Lee HS, et al. MET in gastric carcinomas: comparison between protein expression and gene copy number and impact on clinical outcome. Br J Cancer 2012;107:325-333.

17 Amemiya H, Kono K, Itakura J, et al. c-Met expression in gastric cancer with liver metastasis. Oncology 2002;63:286-296.
18 Drebber U, Baldus SE, Nolden B, et al. The overexpression of c-met as a prognostic indicator for gastric carcinoma compared to p53 and p21 nuclear accumulation. Oncol Rep 2008;19:1477-1483.

19 Heideman DA, Snijders PJ, Bloemena E, et al. Absence of tpr-met and expression of c-met in human gastric mucosa and carcinoma. J Pathol 2001;194:428-435.

20 Huang TJ, Wang JY, Lin SR, et al. Overexpression of the c-met protooncogene in human gastric carcinomacorrelation to clinical features. Acta Oncol 2001;40: 638-643.

21 Wu CW, Li AF, Chi CW, et al. Hepatocyte growth factor and Met/HGF receptors in patients with gastric adenocarcinoma. Oncol Rep 1998;5:817-822.

22 Yonemura Y, Kaji M, Hirono Y, et al. Correlation between overexpression of c-met gene and the progression of gastric cancer. Int J Oncol 1996;8:555-560.

23 Toiyama Y, Yasuda H, Saigusa S, et al. Co-expression of hepatocyte growth factor and c-Met predicts peritoneal dissemination established by autocrine hepatocyte growth factor/c-Met signaling in gastric cancer. Int J Cancer 2011;130:2912-2921.

24 Janjigian YY, Tang LH, Coit DG, et al. MET expression and amplification in patients with localized gastric cancer. Cancer Epidemiol Biomarkers Prev 2011;20: 1021-1027.

25 Spigel DRET, Ramlau R, Daniel DB, et al. Final efficacy results from OAM4558g, a randomized phase II study evaluating MetMAb or placebo in combination with erlotinib in advanced NSCLC, 2011 ASCO Annual Meeting: Chicago, IL, USA, 2011.

26 Gherardi E, Birchmeier W, Birchmeier C, et al. Targeting MET in cancer: rationale and progress. Nat Rev Cancer 2012;12:89-103.

27 Iveson TDR, Davidenko I, Tjulandin S, et al. Safety and efficacy of epirubicin, cisplatin, and capecitabine (ECX) plus rilotumumab (R) as first-line treatment for unresectable locally advanced (LA) or metastatic (M) gastric or esophagogastric junction (EGJ) adenocarcinoma, European Journal of Cancer. Stockholm, 2011.

28 Bilous M, Dowsett M, Hanna W, et al. Current perspectives on HER2 testing: a review of national testing guidelines. Mod Pathol 2003;16:173-182.

29 Macdonald JS, Smalley SR, Benedetti J, et al. Chemoradiotherapy after surgery compared with surgery alone for adenocarcinoma of the stomach or gastroesophageal junction. N Engl J Med 2001;345:725-730.

30 Hofmann M, Stoss O, Shi D, et al. Assessment of a HER2 scoring system for gastric cancer: results from a validation study. Histopathology 2008;52:797-805.

31 Cho EY, Choi YL, Han JJ, et al. Expression and amplification of Her2, EGFR and cyclin D1 in breast cancer: immunohistochemistry and chromogenic in situ hybridization. Pathol Int 2008;58:17-25.

32 Ruschoff J, Hanna W, Bilous M, et al. HER2 testing in gastric cancer: a practical approach. Mod Pathol 2012;25:637-650.

33 Lennerz JK, Kwak EL, Ackerman A, et al. MET amplification identifies a small and aggressive subgroup of esophagogastric adenocarcinoma with evidence of responsiveness to crizotinib. J Clin Oncol 2011;29:4803-4810. 